\title{
Protection of Transmission Line Based on the Severity Index Using Generation Rescheduling Strategy
}

\author{
Sabah H. Alwan \\ Faculty of Engineering, University of Kerbala, Kerbala 56001, Iraq
}

Corresponding Author Email: sabah77@uokerbala.edu.iq

https://doi.org/10.18280/ejee.210606

Received: 10 June 2019

Accepted: 8 August 2019

\section{Keywords:}

line contingency, transmission line overloading, differential evolution algorithm, generation rescheduling, severity index

\begin{abstract}
The overload of the transmission line is a serious problem in the protection of power systems. To eliminate this problem, it is necessary to keep the transmission network immune to the undesired conditions. This paper offers a special protection plan for power systems based on evolutionary algorithms (EAs). Specifically, the differential evolution (DE) algorithm was selected to optimize the magnitude of the severity index (SI) related to the rescheduling strategy, and thus prevent the transmission line form overloading in contingencies. The effectiveness of the proposed DE-based protection plan was compared with those generated by the genetic algorithm (GA) in the same power system. Two conditions were created for the comparison, namely, the N-1 contingency condition based on the normal case, and the perturbation demand condition. The results show that the proposed protection plan outperformed that generated by the GA in minimum fuel cost, computing speed and convergence. The research results provide a new and effective way to protect power systems against possible overload of the transmission line.
\end{abstract}

\section{INTRODUCTION}

System Protection Scheme denoted by (SPS) is prepared to find out abnormal conditions during the operation of a power system which is based on contingency condition and trying to initiate pre-determined processed actions, not only isolation of faulted elements but also alleviation the consequences of the undesired system situations as well as providing an acceptable performance for a system. These schemes are also defined as Remedial Action Schemes (RASs). RAS actions include the load shedding schemes or load changing (in the demand side), and the generation changing or generation rescheduling (in the generation side). In addition to that, changing of the system arrangement, to handle acceptable voltage and power flow and maintain system stability during the system operation as well [1]. Operating system of protection schemes is carried out via the incidence of a system disturbance like the voltage instability, transient angular instability, frequency instability, as well as other instabilities resulting from cascading line outing [2].

The protection of transmission grid from the congestion risk during critical contingencies is an important issue which needs to be taken into consideration through the designing of SPS schemes. The overloading issue of any transmission grid may emerge from some disturbances such as load disturbances i.e. sudden load increasing or decreasing, transmission line power cut and/or transformer failure. Consequently, this happens when no communication is found between the generation and transmission networks as well [3].

Thus, the overloading issue appeared in any transmission grid could lead to cascading line outage and therefore resulting in a system collapse. Consequently, in order to obviate the system overloading situation, some specific actions have to be taken into consideration. For instance, the generation rescheduling, transmission line switching, phase shift transformers, and load shedding programs [3]. Demand side management (which means that the load is varied according to the contingency condition by the load shedding schemes) as well as the active power rescheduling (i.e. rescheduling of the generation strategy) are the generality utilized SPS responses for alleviating the transmission congestion issue where in which additional reserves are not needed.

The studies of the system impact and also the assessment of the security are in general deal with $\mathrm{N}-1$ contingency term and that implies the absence of one of the system components e.g. generator, transformer, and transmission line without losing of the demand. Line congestion regarding the protection within a system could become a significant task in $\mathrm{N}-1$ contingency state. Thus, it is specified that the system can operate within typical conditions and hence, resists these emergencies without any violations or not [4]. Therefore, the study on the system requires an evaluation of a contingency condition such as the $\mathrm{N}-1$ contingency condition, and in addition to that the situation under the post contingency loading which will be either violates the emergency level or not in order to evaluate the system operating restrictions. Construction of new transmission networks for accommodating the $\mathrm{N}-1$ situation is generally needed an extra cost beside the time-consuming. Hence, applying the generation rescheduling as well as the load shedding techniques illustrate the suitable actions for protecting the power system against the system collapse through a specific critical situation [5].

In this research, the artificial intelligence namely, Differential Evolution (DE) algorithm, was executed in the applied technique special protection scheme on the studied system contingencies. and This algorithm has been utilized in order to relieve the line congestion risk due to the values of the severity index criteria which considered as the objective 
function to decide the optimal magnitudes related to the generation rescheduling process. The validation of the executed methods (i.e. DE \& GA) was evaluated based on some selected contingency cases within the IEEE 30-bus system.

\section{GENERATION RESCHEDULING STRATEGY}

In order to keep any power system operates in a normal and secure situation, the loading area of a transmission network has to be kept within the system certain levels. Overloading issue appeared in any transmission network might take place according to unexpected growing in the power consumption, sudden generator outage due to technical causes and/or line outage. In addition to that reasons, the system transmission congestion may happen resulting from failure of any of system components. Power system collapse and cascade line outage could be happened due to the overloading risk of the transmission network. Subsequently, mitigation of the system abnormal situation is considered as a serious challenge within a reliable operation of the power system. The number of pages for the manuscript must be no more than ten, including all the sections. Please make sure that the whole text ends on an even page. Please do not insert page numbers. Please do not use the Headers or the Footers because they are reserved for the technical editing by editors.

A convenient corrective measure should be taken into consideration at a minimal time without a violation of the system restrictions in order to mitigate the line overloads throughout the security boundaries. One of the most successful and safe approaches that applied to the line overloading alleviation is the rescheduling the power of generation units within an established power system to handle this problem [6].

Alleviation of the line congestion status was executed by using different approaches. The generation rescheduling strategy has been executed based on the cascade back propagation technique within the neural network [7]. The implemented algorithm was used in order to predict the line overloading magnitude as well as to relieve this overloading status due to the N-1 phenomena along with unexpected growing in the demand as well. Sharma and Srivastava [8] have proposed an approach based on cascading neural network in order to specify the network lines which overloaded through the used power system. Furthermore, prediction of the amount of the line overloading in the identified overloaded lines and was tested on IEEE 14-bus system. The utilized approach was supported through various loading and generation conditions.

Optimal generation rescheduling framework via congestion management has been suggested by Dutta and Singh [9]. These techniques were accomplished by using the Particle Swarm Optimization method. The same method has been introduced by Deb and Goswami [10], where this method was used in order to relieve the transmission congestion issue by achieving a strategy based on the generation rescheduling.

The rescheduling has also been applied via the area of the optimal power flow due to the minimizing of the total congestion of the considered network. Hagh and Galvani [11] have been addressed a new version of non-dominated sorting genetic algorithm and this algorithm was addressed as an optimization tool. The proposed technique was executed for determining the amount and position of the network demand to be shed and the amount of the generation rescheduling throughout post contingency. Differential Evolution technique was used for reactive power rescheduling [12], where this algorithm was implemented to enhance a voltage stability. The similar algorithm (i.e. DE) has also been applied in the load shedding domain within a power system [13]. The authors applied DE method for improving the voltage stability framework.

\section{THE RESEARCH METHODOLOGY}

\subsection{The scheme mathematical formulation}

When a power system is subjected to an emergency situation, some analyses need to be taken into account. Throughout the analysis of the system contingency situation, the primary job of the operators is the relieving of line overloading risk. Therefore, in this research work, the objective function is considered as the minimized magnitude related to the severity index. The severity minimization challenges as well as the rescheduling strategy for the active power generation are subjected to the constraints that associated with any power system along its operation. Thus, the system should follow up these constraints which assorted into two groups and its details as following:

\subsubsection{Equality constraints}

Where this section of constraints comprise the load flow constraints that showed as:

$$
\begin{aligned}
& P_{i}=V_{i} \sum_{j=1}^{N B} V_{j}\left(G_{i j} \cos \theta_{i j}+B_{i j} \sin \theta_{i j}\right) \\
& Q_{i}=V_{i} \sum_{j=1}^{N B} V_{j}\left(G_{i j} \sin \theta_{i j}-B_{i j} \cos \theta_{i j}\right)
\end{aligned}
$$

where, Pi, and Qi express the injected real and reactive power to bus $\mathrm{i}$. Gij, and Bij are the self-conductance and susceptance of the line between buses $\mathrm{i}-\mathrm{j}$. $\mathrm{Vi}$, and $\mathrm{Vj}$ represent the voltage value at buses $i$ and $j$ respectively. And $\theta \mathrm{ij}$ is the voltage angle difference between the bus $i$ and $j$.

\subsubsection{Inequality constraints}

These power system constraints express the active and reactive power boundaries, bus voltage working space, as well as the transmission line flow limits:

$$
\begin{gathered}
P_{G i}^{\min } \leq P_{G i} \leq P_{G i}^{\max } \\
Q_{G i}^{\min } \leq Q_{G i} \leq Q_{G i}^{\max }
\end{gathered}
$$

where, $\mathrm{i}=1,2, . . \mathrm{NG}$

$$
\begin{array}{ll}
V_{i}^{\text {min }} \leq V_{i} \leq V_{i}^{\max } & i=1,2, \ldots N B \\
\left|S_{i j}\right| \leq S_{i j}^{\text {max }} & i=1,2, \ldots N L
\end{array}
$$

where, $\mathrm{P}_{\mathrm{Gi}}, \mathrm{Q}_{\mathrm{Gi}}$ denoted to the active and reactive powers found in bus i. Sij represent a line power flow that lies between the buses $i$ and $j$.

$\mathrm{NB}$ is the number of buses included within a power system. $\mathrm{NL}$ is the total number of the transmission lines, and NG represents the total number of the grid generators. 


\subsection{The objective function}

The proposed DE-based program has a fitness function which is implemented in order to evaluate the optimum rescheduling of the generation aspect included in a power system and hence for minimizing the total required cost of the generation package. However, the cost is based on the price bids presented by the network companies specialized with the generation field. The goal of the cost minimizing was executed in line with decreasing of the magnitude of the severity index that resulted in alleviating the transmission network overloading problem in post emergency situation.

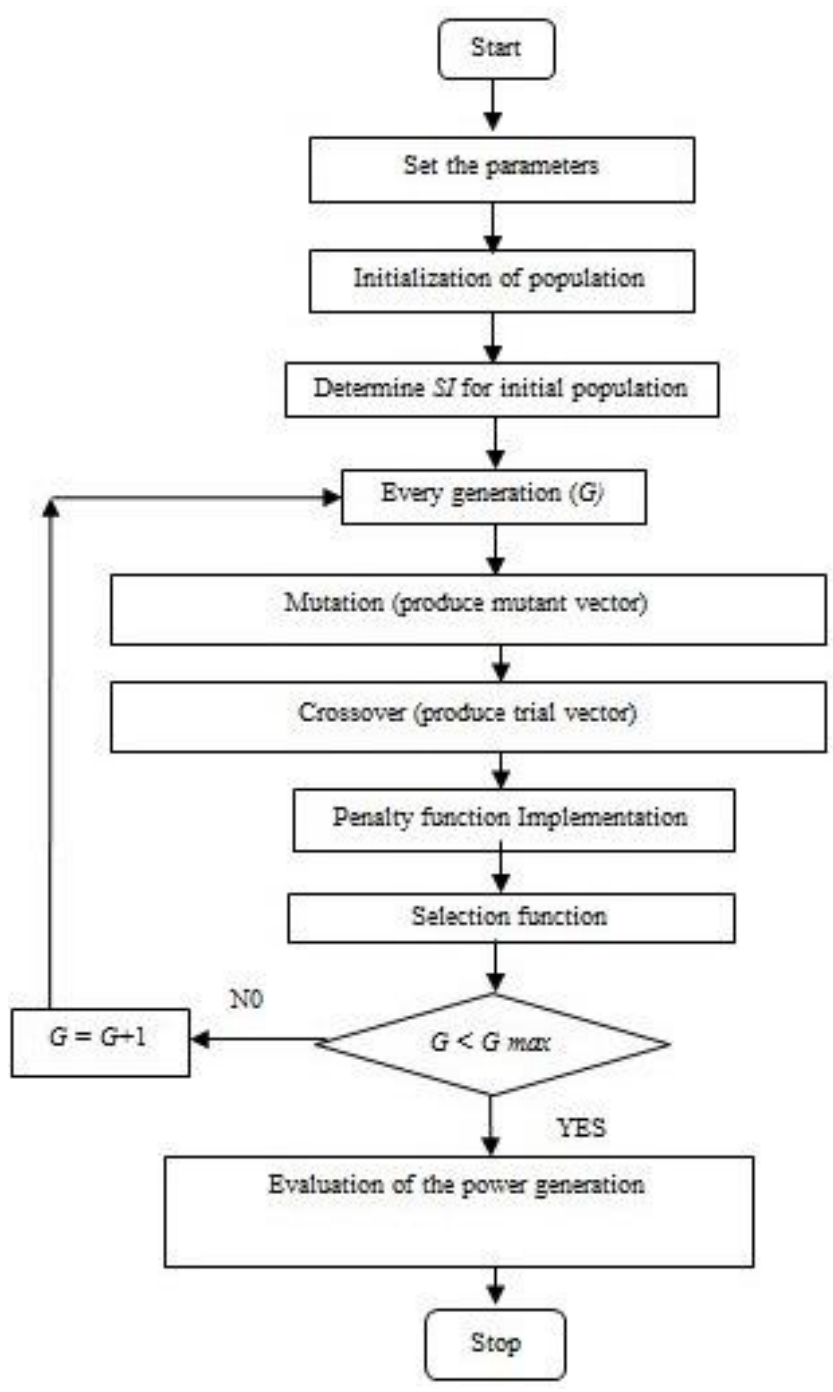

Figure 1. Schematic diagram for the presented scheme

The principle of minimizing the total fuel cost is illustrated by the formula below where the generation cost is subjected to the system constraints as follows:

$$
\min F C=\sum_{i=1}^{N G}\left(a_{i}+b_{i} P_{G i}+c_{i} P_{G i}^{2}\right) \quad \$ / h r
$$

where, FC assigns to the total system generation cost for the normal operation as well as for overloading management. a i, b_i and c_i denoted to the coefficients of the cost and their magnitudes mentioned by Devaraj and Yegnanarayana [4], and Alsac and Stott [14], P_Gi represents the actual power generated by a generator placed in bus $\mathrm{i}$. The entire procedure of the DE based algorithm has been illustrated in the Figure 1.

\section{SEVERITY INDEX (SI)}

The severity index formulation is denoted to the severity of a contingency condition to any power system, where this index is associated with the line overloading risk due to the stress that subjected to a power system within a post contingency situation. This index can be shown as: $[4,7]$.

$$
S I=\sum_{k=1}^{o v l}\left(\frac{s_{i j}}{s_{i j}^{m a x}}\right)^{2 m}
$$

where, $S_{i j}$ is the line flow and can be obtained from the load flow analysis which is subjected to the given equality and inequality constraints. $S_{i j}^{\max }$ expresses the line flow limit, and ovl represents only the set of the overloaded lines that associated with a contingency condition. This study is considered the magnitude of $\mathrm{m}$ has been fixed to 1 . In order to avoid the masking effects during the implementation of the algorithms and for the security assessment, the overloaded lines are considered only while computing the severity magnitudes [7]. Minimization of this index has been undertaken as the objective function to ensure the case that no more lines to be overloaded after executing the generation rescheduling strategy. In order to illustrate a secure situation, the magnitude of the index must be equal to zero and that means no overloaded lines within a system. The greater value of SI, resulted in more critical contingency would be.

\section{SPECIAL PROTECTION AND CONTROL SCHEME BASED ON DIFFERENTIAL EVOLUTION STRATEGY}

Differential evolution algorithm is an evolutionary algorithm that considered as a population based stochatic search technique with a high performance optimization that is easy to execute and understand. Storn and Price introduced DE algorithm in 1997 [15]. This algorithm suggests a solution to a problem by enhancing the offered solutions iteratively according to a predefined criteria. DE uses real number representation and its optimization procedure is the same as of the GA strategy but unlike simple GA that generally dependent on the crossover operator. At first, DE algorithm initializes a population, then executes the mutation also called differential, crossover, and finally the selection operation to achieve the required process by suggest possible solutions toward a specific issue.

DE initially works on the initializing of a population set that called (P) which includes a candidate solution to a predefined issue. Like other evolutionary techniques, where these solutions called individuals are initially randomly generated then improved iteratively along with mutation, crossover, as well as the selection operators respectively. This process is continue repeating over a number of iterations chosen by a designer until the stopping criteria is reached. This criteria is prespecified as a max number of generations denoted as $\left(G_{\max }\right)$ or required fitness function. The population set which denoted by (P) involves multiple NP-D dimentional real valued individual vectors $\left(\mathrm{X}_{\mathrm{i}, \mathrm{G}}\right)$ shown by $[16]$ :

$$
\begin{gathered}
P_{X, G}=X_{i, G} \\
i=1, \ldots N P, \quad G=1,2 \ldots \ldots G_{\max }
\end{gathered}
$$




$$
\begin{aligned}
& X_{i, G}=X_{j, i, G} \\
& j=1, \ldots \ldots D
\end{aligned}
$$

where, each of the individual vector involves an index that is expressed by the letter $i$ with the range from 1 to NP which considered as the population size. G represents the number of current iterations. The parameter belongs to a vector indexed by the letter $\mathrm{j}$ with its range between 1 and $\mathrm{D}$. In general, $\mathrm{D}$ is considered as the number of control variables included within a specific problem to be solved. In this research, D is equal to the number of generation units located in a power system.

Following are the basic steps included through the DE algorithm as follows:

\subsection{Initialization}

The optimization process of the differential evolution algorithm begins with the initialization stage, means at $\mathrm{t}=\mathrm{O}$. In this stage, an initial population is constructed that includes NP D-dimentional real valued individual vectors denoted by $\mathrm{X}_{\mathrm{i}, \mathrm{G}}=\left[\mathrm{X}_{1, \mathrm{i}, \mathrm{G}}, \mathrm{X}_{2, \mathrm{i}, \mathrm{G}}, \ldots . . \mathrm{X}_{\mathrm{j}, \mathrm{i}, \mathrm{G}} \ldots . . \mathrm{X}_{\mathrm{D}, \mathrm{i}, \mathrm{G}}\right]$, where these vectors are generated and each of them considers a candidate solution to a specific optimization problem.

The parameters related to the optimization process contain lower limits expressed by $\mathrm{XL}=\mathrm{X}_{1, \mathrm{~L}}, \mathrm{X}_{2, \mathrm{~L}}, \ldots ., \mathrm{X}_{\mathrm{D}, \mathrm{L}}$ as well as the upper limits denoted by $\mathrm{X}_{\mathrm{H}}=\mathrm{X}_{1, \mathrm{H}}, \mathrm{X}_{2, \mathrm{H}}, \ldots . ., \mathrm{X}_{\mathrm{D}, \mathrm{H}}$. These lower and upper limits involve the search region belongs to the DE algorithm. The initial jth components of the ith individual vector included through the population set can be initialized by a strategy shown as:

$$
X_{j, i}(t=0)=X_{j, L}+\operatorname{rand}\left(X_{j, H}-X_{j, L}\right)
$$

where, $\mathrm{X}_{\mathrm{j}, \mathrm{i}}$ is the individual vector. $\mathrm{X}_{\mathrm{j}, \mathrm{L}}$ expresses the lower limit and $X_{j, H}$ is the upper limit for each vector. rand is a random number distributed within 0 and 1 .

\subsection{Mutation}

After achieving the initialization process based on the generation of initial vectors, the algorithm produces a new version of vectors based on each initialized individual vector within the population set for each iteration. The new version of the individual vector is called a mutant (or donor) vector $\mathrm{V}_{\mathrm{i}, \mathrm{G}}$ generated based on a strategy designed by adding the difference (which is weighted by a factor named as a mutation factor) between any two randomly chosen vectors to a third one and all the chosen vectors are under the current iteration that shown by the formula below:

$$
V_{i, G}=X_{r 1, G}+F\left(X_{r 2, G}-X_{r 3, G}\right)
$$

The selected vectors have to be distinct from the main target vectors $\mathrm{X}_{\mathrm{j}, \mathrm{i}, \mathrm{G}}$, for which the three randomly selected vectors among the population set denoted by $\left(\mathrm{Xr}_{1, \mathrm{G}}, \mathrm{Xr}_{2, \mathrm{G}}, \mathrm{Xr}_{3, \mathrm{G}}\right)$ are placed within the range from 1 to NP. $r_{1} \neq r_{2} \neq r_{3} \in[1, \ldots . N P]$ refer to randomly generated integers by using a random number generation function. $\mathrm{F}$ is considered as the mutation factor that selected to be in the range between 0 and 1 .

\subsection{Crossover}

After obtaining the mutant vector $\mathrm{V}_{\mathrm{i}, \mathrm{G}}$, the crossover operation introduces in order to rise the diversity for the population set. In this point, the donor (also called mutant) vector $\mathrm{V}_{\mathrm{i}, \mathrm{G}}$ as well as the target vector $\mathrm{X}_{\mathrm{j}, \mathrm{i}, \mathrm{G}}$, are both utilized for creating a new vector called a trial vector indicated by $\mathrm{U}_{\mathrm{j}, \mathrm{i}, \mathrm{G}}$ by the following strategy:

$$
U_{j, i, G}= \begin{cases}V_{j, i, G} & \text { if }\left(\text { rand } \leq \text { CR or } j=j_{\text {rand }}\right) \\ X_{j, i, G} & \text { otherwise }\end{cases}
$$

where, $V_{j, i, G}$ and $X_{j, i, G}$ are aforementioned donor and target vesctors respectivily. CR symbolizes to a parameter named as the crossover factor for DE algorithm and its value is chosen between 0 and 1 . CR controls diversity of the population in addition to support the algorithm in order to away from the local optimum. jrand $\in[1, \ldots . . \mathrm{D}]$, expresses a randomly chosen index to ensure that the trial vector gets at least one element from the donor vector in order to improve its candidate solution.

When the crossover process is accomplished, a penalty function planning is used, where this function is taken into consideration in order to ensure that the final vector magnitudes are through the boundaries after applying both operators, the mutation and crossover respectively based on the following criteria:

$$
U_{j, i, G}=X_{j, i, L}+\operatorname{rand}\left(X_{j, i, H}-X_{j, i, L}\right)
$$

\subsection{Selection}

It is the final process within the DE algorithm and it is undertaken for keeping the population size as a constant form. A selection stage is executed in order to find out either the trial vector $U_{i, G}$ or the target vector $X_{i, G}$ will survive to the next iteration (i.e. $\mathrm{G}=\mathrm{G}+1$ ) through the algorithm. Therefore, this operation can be described by the following criteria:

$$
X_{i, G+1}=\left\{\begin{array}{lll}
U_{i, G} & \text { if } \quad J\left(U_{i, G}\right)<J\left(X_{i, G}\right) \\
X_{i, G} & \text { otherwise }
\end{array}\right.
$$

where, $X_{i, G+1}$ represents the vector that will be withdrawed to the following generation. In general, $\mathrm{J}(\mathrm{X})$ demonstrates the fitness function magnitude for the implemented approach which requires to be minimized as possible as to achieve the target from implementing the proposed algorithm. Thus, if the fitness magnitude related to the trial vector is lower than the fitness that related to the target vector, then it exchanges the corresponding target vector fitness with the trial vector fitness and transferred to the next generation, else the target magnitude is survived. Hence, after performing this strategy, the population set will be better or stays fixed especially for the fitness patterns.

\section{GENERAL}

The implementation of the presented research algorithm was validated on the IEEE 30-bus system. This validation was for showing the effectiveness associated with the executed DE based SPCS scheme. In addition to that, the findings of DE approach are compared with Genetic Algorithm results in terms of the fuel generation cost as well as the speed of fitness convergence. The both executed algorithms have been established within the Matlab environment. The system data corresponding to this work which are undertaken adopted from 
the references $[14,17]$ regarding the parameters related to the grid transmission line as well as the generation operating limits and constraints. The data regarding to the executed system for the total active and reactive powers related to the demand are 283.4 MW and 126.2 MVAR respectively. The load flow analysis was carried out, particularly the Newton - Raphson method for evaluating the parameters data corresponding to each bus within the given power system that includes some rating, specifically: voltage magnitude via its phase angle, in addition to the active and reactive power flows. Moreover, the parameters concerning to the grid lines: line losses and active and reactive power as well. Throughout a power system operation, the line overloads may occur according to several causes containing the case of the single line outage. Hence, the $\mathrm{N}-1$ contingency analyses have been implemented under the base case in addition to the increased load situations in order to characterize the prospective contingencies throughout the running of the power system.

\subsection{Analysis of system contingency conditions}

When the system designers attempt to plan the protection schemes related to power systems, the system contingency analysis should be taken into consideration. Therefore, this pattern is performed under the base as well as the increased load demand conditions in this field of study. Where the analysis is done in order to evaluate the most harmful disturbances in specific conditions assigned by the designer through a power system. The load flow calculations have been used, where the Newton-Raphson method is executed in this work on the IEEE 30 bus system. This method is implemented in pre and post contingency conditions for obtaining the line flows. The resulted line flow is compared with the standard line limit in order to characterize which transmission line gets overloaded due to the identified scenario of the accomplished single line outage based on the severity index. Thus, N-1 contingency situations are implemented under the base and increased demand by $10 \%$ situations. In this study, the limits related to each line flow are considered through the both utilized algorithms and undertaken from [14]. The line outages number as $1-2,1-3,3-4$, and $2-5$, where the first number is the sending bus and the second is the receiving bus, have resulted in overloading some of the other running network transmission lines within the given system under the normal load condition since these affected lines have already exceeded their allowable limits. Thus, the line outage details of the simulated cases before implementing the undertaken generation rescheduling strategy along with its related overloaded lines are shown in Table 1. This table also includes the magnitudes of the SI related to every case study based on the aforementioned severity index criteria. In the increased load situations, the overall active and reactive power consumed within the system have increased from 283.4 MW to 311.74 MW and from 126.2 MVar to 138.82 MVar respectively. The line outage details before the generation rescheduling scheme are also showed in the Table 2 for the increased demand cases.

Table 1. Executed line outages under base load before generation rescheduling strategy

\begin{tabular}{|c|c|c|c|c|}
\hline $\begin{array}{c}\text { Line } \\
\text { outage }\end{array}$ & $\begin{array}{c}\text { lines } \\
\text { overloaded }\end{array}$ & $\begin{array}{c}\text { Line } \\
\text { flow } \\
\text { (MVA) }\end{array}$ & $\begin{array}{c}\text { Flow limit } \\
\text { (MVA) }\end{array}$ & SI \\
\hline $1-2$ & $1-3$ & 307.803 & 130 & 16.265 \\
\hline
\end{tabular}

\begin{tabular}{|c|c|c|c|c|}
\hline & $3-4$ & 279.121 & 130 & \\
\hline & $4-6$ & 174.058 & 90 & \\
& $6-8$ & 36.362 & 32 & \\
\hline $1-3$ & $1-2$ & 273.019 & 130 & 9.279 \\
\hline & $2-4$ & 86.154 & 65 & \\
\hline & $2-6$ & 92.759 & 65 & \\
& $6-8$ & 33.188 & 32 & \\
\hline $3-4$ & $1-2$ & 270.07 & 130 & 9.076 \\
\hline & $2-4$ & 84.916 & 65 & \\
\hline & $2-6$ & 91.805 & 65 & \\
\hline $2-5$ & $6-8$ & 32.928 & 32 & \\
\hline & $1-2$ & 164.467 & 130 & 10.885 \\
\hline & $2-4$ & 74.604 & 65 & \\
\hline & $2-6$ & 102.858 & 65 & \\
\hline & $4-6$ & 124.097 & 90 & \\
& $5-7$ & 110.189 & 70 & \\
& $6-8$ & 33.317 & 32 & \\
\hline
\end{tabular}

Table 2. Executed line outages under increased load before generation rescheduling strategy

\begin{tabular}{|c|c|c|c|c|}
\hline Line outage & $\begin{array}{c}\text { lines } \\
\text { overloaded }\end{array}$ & $\begin{array}{c}\text { Line } \\
\text { flow } \\
\text { (MVA) }\end{array}$ & $\begin{array}{c}\text { Flow } \\
\text { limit } \\
\text { (MVA) }\end{array}$ & \\
\hline $\mathbf{1 - 2}$ with load & $1-3$ & 369.586 & 130 & 22.580 \\
increased by & $2-4$ & 77.239 & 65 & \\
\cline { 2 - 5 } $\begin{array}{c}10 \% \text { at all } \\
\text { buses }\end{array}$ & $3-4$ & 321.795 & 130 & \\
\cline { 2 - 5 } & $4-6$ & 201.235 & 90 & \\
\hline 3-4 with load & $6-8$ & 44.791 & 32 & \\
\cline { 2 - 5 } increased by & $1-2$ & 305.287 & 130 & 11.518 \\
\cline { 2 - 5 } $10 \%$ at all \\
buses & $2-4$ & 93.888 & 65 & \\
\cline { 2 - 5 } & $2-6$ & 101.556 & 65 & \\
& $6-8$ & 38.874 & 32 & \\
\hline
\end{tabular}

\subsection{The performed algorithms}

The power flow within any transmission grid must not exceed the permissible levels for both the normal and abnormal situations in order to achieve a secure and optimal power system operation to make the customer being delivered by continuous electricity. Therefore, an appropriate corrective action might be taken into consideration for alleviation the transmission network overloads. The objective idea behind this work is the alleviation of the line overloading risk by selection the optimal power generated using the generation rescheduling strategy through the contingency analysis. This strategy has been utilized by determining the optimal magnitudes of the generation based on the differential evolution algorithm.

Generated powers from the generation units positioned inside the selected power system are chosen as the control variables of the carried out methods (i.e. DE and GA), where the outcomes resulted from the DE algorithm are compared with those results from the application of the GA for the similar case studies. The number of the generation units in the selected test system are six placed on the buses numbered as $1,2,5,8,11$, and 13 . Initially (i.e. the initialization stage), set of values assigned by the individual vectors are randomly build by efficient DE mechanism within the specified limits (i.e. equality and inequality limits that mentioned earlier), such that the equation (3) is satisfied through the min and max limits. After this step, the DE executes these suggested PG sets inside the fitness function algorithm for getting the resulted severity index values. Subsequently, DE algorithm applies its next strategies such as the mutation as well as the crossover to gain a less and better fitness values for each individual vector. The 
parameters related to DE and GA algorithms need to be regulated. The parameter settings of $\mathrm{DE}$ based scheme are as follows: the crossover factor $(\mathrm{CR})$ and the mutation factor $(\mathrm{F})$ are chosen 0.5 and 0.8 respectively. However, the crossover probability is selected as 0.8 and the mutation probability is 0.3 for GA approach since these values present good results according to several trials. The load flow analysis has been applied in order to acquire the running line flows and as a consequence determining the severity index. The objective function of the proposed research work is considered as the minimized severity magnitudes. The suggested rescheduling values for the generation units within the system by the DE algorithm, can guide us to acquire the minimized costs of the generation based on the minimized severity values. The suggested active powers for each generator within the selected power system for both algorithms are shown in Table 3 along with the particular line contingencies under the given normal demand. The generation costs related to each case study are also shown in Table 3, where this table appears that the DE based method offers less generation cost than the cost presented by GA technique. The overall losses associated with each simulated case are also shown.

Table 3. Control variables setting under base load

\begin{tabular}{|c|c|c|c|c|c|c|c|c|c|}
\hline Approach & line out of service & \multicolumn{8}{|c|}{ Generated Power $(\mathbf{M W})$} \\
\cline { 2 - 10 } & & $\mathrm{P}_{1}$ & $\mathrm{P}_{2}$ & $\mathrm{P}_{5}$ & $\mathrm{P}_{8}$ & $\mathrm{P}_{11}$ & $\mathrm{P}_{13}$ & System Losses $(\mathrm{mw})$ & Cost $(\$ / \mathrm{hr})$ \\
\hline \multirow{3}{*}{$\mathrm{NE}$} & $1-2$ & 124.87 & 46.12 & 41.53 & 30.97 & 20.19 & 32.97 & 12.82 & 880.96 \\
\cline { 2 - 10 } & $1-3$ & 128.65 & 42.75 & 39.81 & 31.18 & 20.41 & 29.18 & 8.30 & 854.93 \\
\cline { 2 - 10 } & $3-4$ & 129.07 & 42.62 & 35.31 & 30.81 & 21.02 & 32.61 & 7.92 & 846.79 \\
\cline { 2 - 10 } & $2-5$ & 149.59 & 40.37 & 32.69 & 24.46 & 21.13 & 28.97 & 13.32 & 848.67 \\
\hline \multirow{3}{*}{ GA } & $1-2$ & 126.77 & 43.51 & 42.27 & 27.50 & 22.48 & 33.81 & 12.94 & 882.74 \\
\cline { 2 - 9 } & $1-3$ & 127.65 & 42.97 & 39.31 & 28.64 & 21.59 & 31.60 & 8.28 & 855.91 \\
\cline { 2 - 9 } & $3-4$ & 129.99 & 42.16 & 34.74 & 29.34 & 21.98 & 33.40 & 8.11 & 847.04 \\
\cline { 2 - 9 } & $2-5$ & 144.10 & 38.35 & 30.47 & 28.79 & 22.83 & 32.40 & 13.39 & 852.85 \\
\hline
\end{tabular}

Table 4. Control variables setting under increased load

\begin{tabular}{|c|c|c|c|c|c|c|c|c|c|}
\hline \multirow{2}{*}{ Approach } & line out of service & \multicolumn{9}{|c|}{ Generated Power (MW) } \\
\cline { 2 - 10 } & & $\mathrm{P}_{1}$ & $\mathrm{P}_{2}$ & $\mathrm{P}_{5}$ & $\mathrm{P}_{8}$ & $\mathrm{P}_{11}$ & $\mathrm{P}_{13}$ & System Losses (mw) & Cost $(\$ / \mathrm{hr})$ \\
\hline \multirow{2}{*}{$\mathrm{DE}$} & $1-2$ & 126.78 & 65.17 & 46.70 & 31.71 & 21.97 & 33.50 & 14.06 & 1003.17 \\
\cline { 2 - 10 } & $3-4$ & 133.14 & 55.34 & 45.62 & 32.25 & 20.77 & 36.46 & 11.83 & 988.08 \\
\hline \multirow{2}{*}{$\mathrm{GA}$} & $1-2$ & 130.03 & 60.15 & 47.18 & 31.25 & 24.01 & 33.92 & 14.75 & 1004.99 \\
\cline { 2 - 9 } & $3-4$ & 136.13 & 47.51 & 47.77 & 32.34 & 22.85 & 36.22 & 11.09 & 991.67 \\
\hline
\end{tabular}

Table 5. Line details after generation rescheduling scheme for base load cases

\begin{tabular}{|c|c|c|c|c|c|}
\hline Line out of service & System lines & Flow of line (MVA) (DE) & Flow of line (MVA) (GA) & Line limit (MVA) & $S I$ \\
\hline \multirow[t]{4}{*}{$1-2$} & $\begin{array}{l}1-3 \\
2-4\end{array}$ & $\begin{array}{c}123.144 \\
24.384\end{array}$ & $\begin{array}{c}125.732 \\
25.41\end{array}$ & $\begin{array}{c}130 \\
65\end{array}$ & 0 \\
\hline & $3-4$ & 116.283 & 118.754 & 130 & \\
\hline & $4-6$ & 73.506 & 75.477 & 90 & \\
\hline & $6-8$ & 13.152 & 13.304 & 32 & \\
\hline \multirow[t]{4}{*}{$1-3$} & $1-2$ & 128.068 & 127.244 & 130 & 0 \\
\hline & $2-4$ & 44.752 & 44.245 & 65 & \\
\hline & $2-6$ & 47.477 & 47.251 & 65 & \\
\hline & $6-8$ & 7.040 & 7.850 & 32 & \\
\hline \multirow[t]{4}{*}{$3-4$} & $1-2$ & 126.559 & 127.384 & 130 & 0 \\
\hline & $2-4$ & 42.758 & 42.677 & 65 & \\
\hline & $2-6$ & 46.112 & 46.163 & 65 & \\
\hline & $6-8$ & 8.085 & 8.422 & 32 & \\
\hline \multirow[t]{6}{*}{$2-5$} & $1-2$ & 85.544 & 83.127 & 130 & 0 \\
\hline & $2-4$ & 43.368 & 40.128 & 65 & \\
\hline & $2-6$ & 59.528 & 55.383 & 65 & \\
\hline & $4-6$ & 71.591 & 67.608 & 90 & \\
\hline & $5-7$ & 69.689 & 68.666 & 70 & \\
\hline & $6-8$ & 12.509 & 12.777 & 32 & \\
\hline
\end{tabular}

The performed algorithms (i.e. DE \& GA) are executed for 50 iterations, and simulated at least 10 individual runs. Consequently, the generation magnitudes are considered as the average for the overall individual runs. Table 4 illustrates the control variables under the load perturbation situation with the detailed generated values in addition to the generation magnitudes. The overloaded line details after applying the undertaken schemes are shown in Table 5 for the base load cases, where in this table, the presented power is completely mitigated the associated overloaded grid lines due to the new severity levels that has been totally reached to zero. This is indicated that no more lines get overloaded after applying the research strategy (i.e. the generation rescheduling) scheme. The line details after the rescheduling scheme are illustrated in Table 6 for the increased load conditions. Figure 2 shows the DE performance regarding the optimal magnitudes of the severity index for 50 generations which considered as the objective function for the utilized protection scheme to handle 
the considered line outage scenarios such as 1-2, 1-3, 3-4, and $2-5$. For these resulted values of the severity index, the algorithm will decide the magnitudes of the generation rescheduling It can be seen from the figure that the DE algorithm has fast fitness convergence within the first ten iterations for all applied case studies.

Table 6. Line details after generation rescheduling scheme for increased load cases

\begin{tabular}{|c|c|c|c|c|c|}
\hline $\begin{array}{c}\text { Line out } \\
\text { of service }\end{array}$ & $\begin{array}{c}\text { System } \\
\text { lines }\end{array}$ & $\begin{array}{c}\text { Flow of } \\
\text { line } \\
\text { (MVA) } \\
\text { (DE) }\end{array}$ & $\begin{array}{c}\text { Flow of } \\
\text { line } \\
\text { (MVA) } \\
\text { (GA) }\end{array}$ & $\begin{array}{c}\text { Line } \\
\text { limit } \\
\text { (MVA) }\end{array}$ & SI \\
\hline $1-2$ & $1-3$ & 124.888 & 127.852 & 130 & 0 \\
\hline & $2-4$ & 21.141 & 22.733 & 65 & 0 \\
\hline & $3-4$ & 117.563 & 120.385 & 130 & \\
\hline $3-4$ & $4-6$ & 76.224 & 77.336 & 90 & \\
\hline & $6-8$ & 9.732 & 9.919 & 32 & \\
\hline & $1-2$ & 127.499 & 128.977 & 130 & 0 \\
\hline & $2-4$ & 47.139 & 45.643 & 65 & \\
\hline & $2-6$ & 50.865 & 49.087 & 65 & \\
\hline
\end{tabular}

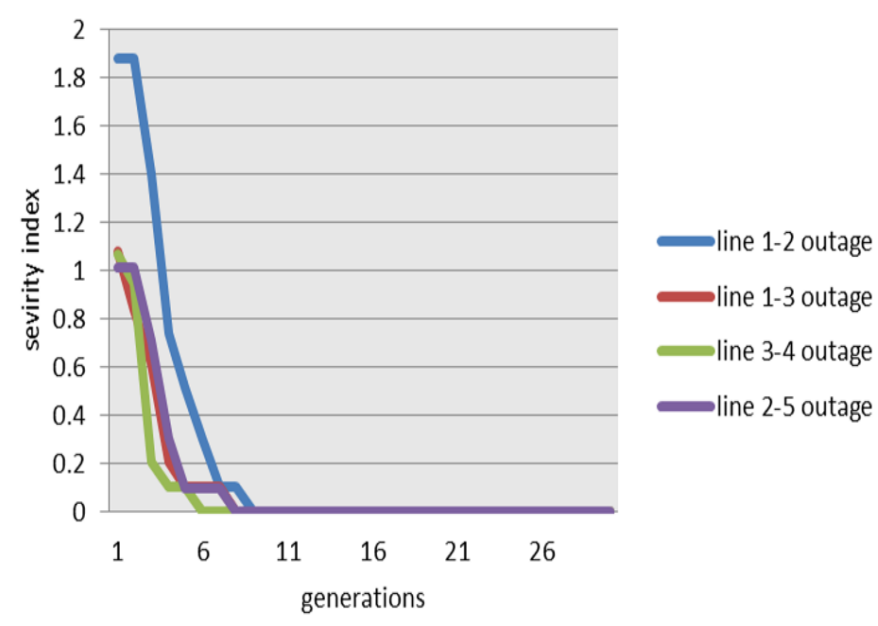

Figure 2. Fitness convergence of DE algorithm

\section{CONCLUSIONS}

The important matter of this paper is designing an SPCS scheme based on the DE algorithm for solving the transmission line overloads problem. The undertaken implemented strategy is fully relieved and solved the grid line overloads risk on the form of a Special Protection Scheme that including the generation rescheduling strategy throughout the minimization of the severity index that considered as a preventive control scheme. The line overloading risk represented by an unexpected outage of a network line called $\mathrm{N}-1$ contingency condition that means the line is out of service resulted from an external effect under the base and the increased load situations is considered in the area of this study. IEEE 30-bus system has been utilized to prove the worthiness of the executed schemes. In order to validate the study findings, $\mathrm{DE}$ results have been investigated with those results from GA simulation. Based on the outcomes, the algorithm of the DE based scheme gives computationally minimum generation cost than GA as well as its performance was closer to the minimized severity index magnitudes that means faster in terms of the speed of convergence. This implies the robustness in case of the minimized generation cost and fast decision making associated with DE approach from its implementation on the identified system contingency conditions.

\section{ACKNOWLEDGMENT}

The author would like to thank the reviewers for their constructive comments, which boosted the work offered in this paper.

\section{REFERENCES}

[1] Cholley, P., Crossley, P., Van Acker, V., Van Cutsem, T., Fu, W., Soto Idia Òez, J., Piekutowski, M. (2001). System Protection Schemes in power networks. CIGRE Technical Brochure.

[2] Othman, M.L., Aris, I., Wahab, N.I.A. (2014). Modeling and simulation of the industrial numerical distance relay aimed at knowledge discovery in resident event reporting. Simulation, 90(6): 660-686. https://doi.org/10.1177/0037549714532961

[3] Awais, M., Basit, A., Adnan, R., Khan, Z.A., Qasim, U., Shafique, T., Javaid, N. (2015). Overload management in transmission system using particle swarm optimization. Procedia Computer Science, 52: 858-865. https://doi.org/10.1016/j.procs.2015.05.143

[4] Devaraj, D., Yegnanarayana, B. (2005). Geneticalgorithm-based optimal power flow for security enhancement. IEE Proceedings-Generation, Transmission and Distribution, 152(6): 899-905. https://doi.org/10.1049/ip-gtd:20045234

[5] Talukdar, B.K., Sinha, A.K., Mukhopadhyay, S., Bose, A. (2005). A computationally simple method for costefficient generation rescheduling and load shedding for congestion management. International Journal of Electrical Power \& Energy Systems, 27(5-6): 379-388. https://doi.org/10.1016/j.ijepes.2005.02.003

[6] Pandiarajan, K., Babulal, C.K. (2014). Transmission Line Management Using Hybrid Differential Evolution with Particle Swarm Optimization. Journal of Electrical Systems, 10(1): 21-35.

[7] Balaraman, S., Kamaraj, N. (2012). Cascade BPN based transmission line overload prediction and preventive action by generation rescheduling. Neurocomputing, 94 : 1-12. https://doi.org/10.1016/j.neucom.2012.04.022

[8] Sharma, S., Srivastava, L. (2008). Prediction of transmission line overloading using intelligent technique. Applied Soft Computing, 8(1): 626-633. https://doi.org/10.1016/j.asoc.2007.05.001

[9] Dutta, S., Singh, S.P. (2008). Optimal rescheduling of generators for congestion management based on particle swarm optimization. IEEE transactions on Power Systems, 23(4): 1560-1569. https://doi.org/10.1109/TPWRS.2008.922647

[10] Deb, S., Goswami, A.K. (2012). Mitigation of congestion by generator rescheduling using particle swarm optimization. 2012 1st International Conference on Power and Energy in NERIST (ICPEN), pp. 1-6. https://doi.org/10.1109/ICPEN.2012.6492332

[11] Hagh, M.T., Galvani, S. (2010). A multi objective genetic algorithm for weighted load shedding. 2010 18th Iranian Conference on Electrical Engineering, pp. 867- 
873.

https://doi.org/10.1109/IRANIANCEE.2010.5506954

[12] Basu, M. (2016). Quasi-oppositional differential evolution for optimal reactive power dispatch. International Journal of Electrical Power \& Energy Systems, 78:

$29-40$ https://doi.org/10.1016/j.ijepes.2015.11.067

[13] Arya, L.D., Singh, P., Titare, L.S. (2012). Differential evolution applied for anticipatory load shedding with voltage stability considerations. International Journal of Electrical Power \& Energy Systems, 42(1): 644-652. https://doi.org/10.1016/j.ijepes.2012.04.006

[14] Alsac, O., Stott, B. (1974). Optimal load flow with steady-state security. IEEE Transactions on Power Apparatus and Systems, 93(3): 745-751. https://doi.org/10.1109/TPAS.1974.293972
[15] Storn, R., Price, K. (1997). Differential evolution-a simple and efficient heuristic for global optimization over continuous spaces. Journal of global optimization, 11(4):

341-359. https://doi.org/10.1023/A:1008202821328

[16] Singh, H., Srivastava, L. (2014). Modified differential evolution algorithm for multi-objective VAR management. International Journal of Electrical Power \& Energy Systems, 55: 731-740. https://doi.org/10.1016/j.ijepes.2013.10.015

[17] Lee, K.Y., Park, Y.M., Ortiz, J.L. (1985). A united approach to optimal real and reactive power dispatch. IEEE Transactions on Power Apparatus and Systems, 104(5):

1147-1153. 